\title{
SOME STUDIES ON THE USE OF Metarhizium anisopliae (METSCH.) SOR. FOR THE CONTROL OF Oryctes rhinoceros $\mathrm{N}$ SRI LANKA
}

\author{
L.C.P Fernando, P. Kanagaratnam ${ }^{2}$ and N.K. Narangoda ${ }^{2}$ \\ Coconut Research Institute of Sri Lanka
}

\begin{abstract}
Black beetle, Oryctes rhinoceros L. larvae and adults were inoculated in the laboratory with three isolates of the fungus, Metarhizium anisopliae (Metsch.) Sor, to screen their virulence against black beetle. All the isolates caused $100 \%$ mortality of larvae and adults when treated with suspensions of $10^{7}$ conidia/ml. Comparatively the local isolate caused a slow death rate in larvae and adults than the other isolates. Fifty grams of the maize inoculum per $0.008 \mathrm{~m}^{2}$ of breeding medium were sufficient to cause $100 \%$ death rate in larvae. In the field, the fungus was capable of disseminating at least $10 \mathrm{~m}$ and the impregnation boxes (infection foci) were attractive to black beetle. It is suggested that $M$. anisopliae could be used as an effective bio-control agent in the integrated management of black beetle.
\end{abstract}

\section{INTRODUCTION}

Black beetle, Oryctes rhinoceros $\mathrm{L}$. is considered a major pest of coconut in Sri Lanka. No single method had proved to be successful in suppressing the pest population. At present integration of mechanical, cultural, chemical methods and use of bio-control agent Baculovirus oryctes are being practised as control measures.

Metarhizium anisopliae was one of the first micro-organisms to be used for the biocontrol of insects (Sorokin, 1883). It has a wide host range, primarily Coleopteran larvae and a wide geographical distribution. Although it has been isolated mostly from insects of Mediterranean or tropical areas because of the relatively high thermal requirements of the fungus (Walstad et al., 1970). M. anisopliae variety major (long spored taxon) is a highly specific pathogen of Oryctes beetles (Ferron et.al, 1972) and is being used in many countries to suppress black beetle populations (Latch \& Falloon, 1976; Wikardi, 1983; Evans, 1985). Although it infects black beeile naturally in Sri Lanka, the disease incidence is as low as $1.75 \%$ in the coconut triangle (unpublished data).

'Present address : 919-644, Main Street, West Hamilion, Ontario L8 SI AI, Canada.

${ }^{2}$ Present address: Dept. of Entomology, University of Queensland, $Q$ 4072, Australla. 
The present preliminary study investigated the virulence of three isolates of the fungus, quantity of the fungus required for the inoculation of larvae and its dissemination in the field with a view to increasing the disease incidence under field conditions.

\section{Experiment 1}

\section{MATERIALS AND METHODS}

The fungal isolates local (LS), 148-83 from France and 137-82 from New Zealand which were isolated from $O$. rhinoceros were tested against larvae of black beetle. Only isolates LS and 148-83 were tested against adults. The cultures were prepared by growing them in plates of Sabouraud-Dextrose-Agar (SDA) until sporulation occurred. Spores were scraped into the buffer, Tween 80 and shaken well to produce a homogeneous suspension. A suspension of $10^{7}$ spores per one $\mathrm{ml}$ was prepared by serial dilution with the buffer. Viability of the spores was determined by incubating three drops of the suspension on a thin layer of SDA for 24 hours.

Third instar black beetle larvae and adults of unknown age were collected from the field due to difficulties in breeding them in the laboratory. The larvae were kept in the laboratory for two weeks to obtain larvae appeared to be free of viral infection. Sixty larvae were inoculated with each isolate by dipping them individually in spore suspensions. The same number of larvae dipped in sterilized water were used as the control. Adult beetles were inoculated by allowing them to swim in the respective suspensions for one minute. Twenty one adults were inoculated with each isolate. Another 21 adults which were allowed to swim in water were used as control.

Inoculated insects were incubated for one day. Larvae were then transferred into covered containers filled with sterilized and moist goat dung. Twenty larvae were kept in each container. Each of seven adults was also transferred to each container containing moist goat dung and split bananas. The larvae were examined on the 7th, 10th, 13th and 16 th day after treatment and dead larvae were removed and stored to ascertain the cause of death. The adults were examined on 4 th, $6 \mathrm{th}, 8 \mathrm{th}, 10 \mathrm{th}$ and 13 th day after treatment. The dead larvae and adults that produced Metarhizium spores were regarded as dead due to fungus.

\section{Experiment 2}

Local isolate of $M$. anisopliae was grown on boiled, sterilized maize grains until sporulation occurred. Sixteen clay pots of $0.008 \mathrm{~m}^{2}$ volume were filled with moist coir dust and twenty black beetle larvae (third instar) were released into each pot. Each of four pots chosen randomly was inoculated with $50 \mathrm{~g}, 100 \mathrm{~g}$ and $150 \mathrm{~g}$ of the inoculum by spreading the maize grains on the medium and covering with a thin layer of coir dust. Four untreated pots were kept as controls. The medium was kept moist through: out the experimental period by adding an equal amount of water to each pot. Pots were examined on the 9th, 13th and 20th day after treatment and dead larvae were removed and counted. 


\section{Experiment 3}

Twenty four square shaped impregnation boxes were constructed in the field using two tiers of one meter long coconut logs and filled with mixture of goat dung and coir dust. Each of six boxes was arranged $10 \mathrm{~m}$ apart in four rows. Randomly chosen twelve boxes that were used as controls were released with each of 45 healthy larvae before the treatment to avoid accidental contamination with any fungus material. To the rest of the boxes each of 45 larvae inoculated with the local isolate of $M$. anisopliae were released. The larvae were inoculated by allowing them to crawl in the maize inoculum. Breeding medium in all the boxes was kept moist by adding water regularly. Observations on the larvae infected by the fungus in each box were recorded on 20, 46 and 75 days after treatment. At each observation 10 healthy larvae were released into control boxes and each of 10 inoculated larvae was released into treated boxes.

\section{Experiment 1}

\section{RESULTS}

Variable number of larvae in each replicate died due to causes other than fungal infection (mainly of virus infection). Therefore, the percentage mortality of dead larvae due to fungus at each period was calculated as a proportion of total larvae dead due to fungus and total live larvae at the end of the experimental period. The mortality caused by each isolate at each period was analysed using analysis of variance. The percentage mortalities of adults were also calculated as done for larvae.

All three isolates were virulent and killed the larvae within 13-16 days after treatment: However, the isolates affected differently in causing mortality in larvae (Table 1). The isolates $137-82$ and $148-83$ killed $96.6 \%$ and $95.7 \%$ of the larvae respectively by the 10th day after treatment. Contrast to these two isolates, the local isolate killed only $81.5 \%$ of the larvae by the 10th day and the death rates were spread over time (4-16 days).

Table 1. The percentage proportional mortality of larvae treated with three isolates of Metarhizium anisopliae at 4, 7, 13 and 16 days after treatment. The significance levels were derived from a analysis of variance test.

\begin{tabular}{|c|c|c|c|c|c|}
\hline \multirow[t]{3}{*}{ Isolate } & \multirow[b]{3}{*}{ 4th } & \multicolumn{4}{|c|}{ Percentage mortality } \\
\hline & & \multirow[b]{2}{*}{ 7th } & \multicolumn{2}{|c|}{ (days after inoculation) } & \multirow[b]{2}{*}{16 th } \\
\hline & & & 10th & 13th & \\
\hline LS & 7.0 & 37.4 & 37.1 & 15.0 & 3.5 \\
\hline $137-82$ & 3.7 & 9.7 & 83.2 & 3.4 & 0.0 \\
\hline $148-83$ & 2.6 & 39.6 & 54.8 & 3.0 & 0.0 \\
\hline Significance & ns & $*$ & ** & * & ns \\
\hline
\end{tabular}

ns - non significant $*-\mathrm{P}<0.5 * *-\mathrm{P}<0.1$ 
Both isolates Local and 148-83 were also highly pathogenic against adult black beetle. The adults that did not die due to age succumbed to the disease and deaths occurred from 8th to 13th day (Table 2).

Table 2. The percentage proportional mortality of adults treated with two isolates of Metarhizium anisopliae at 4, 6, 810 and 13 days after treatment.

\begin{tabular}{lllllll}
\hline \multirow{2}{*}{ Isolate } & & \multicolumn{4}{c}{ Percentage mortality (days after inoculation) } \\
\cline { 2 - 7 } & 4th & 6th & 8th & 10th & 13th \\
\hline LS & 0 & - & 0 & 41.7 & 33.3 & 25.0 \\
$148-83$ & 7.1 & 0 & 50.0 & 28.6 & 14.3 \\
Control & 0 & & 0 & 0 & 6.2 & 0 \\
\hline
\end{tabular}

Experiment 2

There was no relationship between the quantity of inoculum used and the rate of infection (Table 3). All the larvae were dead due to treatments between 13-20 days after inoculation indicating that $50 \mathrm{~g}$ of the inoculum are sufficient to infect larvae in a $0.008 \mathrm{~m}^{2}$ of breeding medium. As the inoculum was spread on the surface of the medium the encountering of fungal spores depended on the movement of the larvae in the medium. The high mortality rate of the larvae in the treatment of $100 \mathrm{~g}$ of inoculum could be due to the above reason.

Table 3. Percentage mortality of larvae treated with three different quantities of inoculum grown on maize grains at each time period (days).

Quantity (g)

Percentage mortality

\begin{tabular}{llll} 
& \multicolumn{3}{c}{ (days after inoculation) } \\
& 9 th & 13th & 20th \\
\hline 50 & 0 & 58.8 & 100.0 \\
100 & 24.4 & 74.2 & 100.0 \\
150 & 3.9 & 59.8 & 100.0 \\
\hline
\end{tabular}

Experiment 3

The fungus has naturally disseminated from the treated boxes to untreated boxes with time. The number of infected larvae and number of boxes contaminated with the fungus increased within the three month period (Table 4) indicating its ability to disseminate naturally to other breeding grounds. The inoculated boxes did not contain infected larvae at all times (Table 4). This may be that the infected cadavers might have been disintegrated at the times of observations and due to absence of live larvae for 
infection. The presence of the black beetle eggs and newly hatched larvae indicated clearly that the impregnation boxes attracted black beetle.

Table 4. Number of live larvae, number of infected larvae and number of pits contained infected larvae in the treated and untreated impregnation boxes at different times after treatment.

\begin{tabular}{lccc}
\hline & \multicolumn{3}{c}{ (Number of days after inoculation) } \\
Treatment \& number & 20 days & 46 days & 75 days \\
\hline Untreated & & & \\
$\quad$ Live larvae & 185 & 90 & 39 \\
Infected larvae & 0 & 8 & 17 \\
Pits with infected larvae & 0 & 3 & 8 \\
Treated & & & \\
Live larvae & 46 & 21 & 25 \\
Infected larvae & 44 & 33 & 10 \\
Pits with infected larvae & 12 & 10 & 7 \\
\hline
\end{tabular}

\section{DISCUSSION}

Variation in the strain virulence has been shown most frequently with $M$. anisopliae (Veen, 1968; Ferron et al., 1972; Latch, 1976). In general, results of the present study showed that local and imported isolates caused total mortality of black beetle in laboratory conditions although there were slight differences in their pathogenicity (Tables 1 \& 2). As the local isolate is found infecting Oryctes naturally in Sri Lanka and is readily available in the field, it could be used in field release programs. Also it is suggested that an introduced isolate is unlikely to be more pathogenic than the one present locally (Latch, 1976).

The natural dissemination of the fungal spores to at least $10 \mathrm{~m}$ from the site of infection and attraction of treated boxes to black beetle for a longer time (Table 4) indicate that such treated boxes could effectively serve as infection foci. Although conidia can survive in the soil for several months (Latch \& Falloon, 1976; Milner \& Lutton, 1976) viability of conidia, the infectivity, disease development and death of larvae and adults depend on many other internal and external factors (Clerk \& Medelin, 1965; Walstad et al., 1970; Latch, 1976; Gottwald \& Tedders, 1982; Vey et al., 1982 and Zimmermann, 1982). In the impregnation boxes the treated larvae did not succumb to the disease as in the laboratory experiments (Table 4). Hence, under field conditions the pathogenicity of an isolate could be reduced considerably. The type of breeding medium also affects the survival of conidia as well as its attractiveness to black beetle. Compost and saw dust allowed better survival of the fungus (Latch \& Falloon, 1976).

The present investigation suggests that $M$. anisopliae could be recommended as a potential biological control agent in the integrated management program of black beetle. As the fungus can survive for a longer period its use especially in permanent breeding 
grounds such as coir dust and saw dust heaps could be considered. Further studies on the climatic conditions affecting the effectiveness of the fungus, suitability of different climatic zones for longer survival of the fungus and increasing the attractiveness of infection foci need to be determined for the development of an effective release program of $M$. anisopliae to control black beetle.

\section{ACKNOWLEDGEMENTS}

The authors wish to thank Mr. D.T Mathes, Head, Biometry Division for the analysis of results and Mrs. C.N.K Rajapakse for valuable suggestions in the manuscript.

\section{REFERENCES}

Clerk, G.C and Medelin, M.F. (1965). The longevity of conidia in three insectparasitizing hyphomycetes. Trans. Brit. Mycol. Soc., 148:193-209.

Evans, H.C. (1985). Report prepared for the Government of the Philippines by the Food and Agriculture Organization of the United Nations on Rhinoceros beetle control in typhoon - affected areas using the entomopathogenic fungus Metarhizium anisopliae. pp. 17.

Ferron, P., Hurpin, B. and Robert, P.H. (1972). Sur la specificite de Metarhizium anisopliae (Metsch.) Sorokin. Entomophaga 17(2) : 165-178.

Gottwald, T.R and Tedders, W.L. (1982). Studies on conidia release by the entomogenous fungi Beauveria bassiana and Metarhizium anisopliae (Deuteromycotina: Hyphomycetes) from adult Pecan weevil (Coleoptera: Curculionidae) cadavers. Environ. Entomol. 11: 1274-1279.

Latch, G.C.M. (1976). Studies on the susceptibility of Oryctes rhinoceros to some entomogenous fungi. Entomophaga. 21(1): 31-38.

Latch, G.C.M. and Falloon, R.E. (1976). Studies on the use of Metarhizium anisopliae to control Oryctes rhinoceros. Entomophaga. 21(1): 39-48.

Milner, R.J and Lutton, G.G. (1976). Metarhizium anisopliae: Survival of conidia in the soil. Proceedings of the 6th International Colloqium on Insect Pathology, Kingston (Canada).

Sorokin, N. (1883). Plant parasites of man and animals as causes of infectious diseases. 2: 268-291. (in Russian).

Veen, K.H. (1968). Recherches sur la maladie due a Metarhizium anisopliae chez le criquet pelerin. Mededelingen Landbouwhogeschool, Wagengingen. 68: 1-77.

Vey, A., Fargues, J and Robert, P. (1982). Histological and ultrastructural studies of factors determining the specificity of pathotypes of the fungus Metarhizium anisopliae for scarabeid larvae. Entomophaga. 27(4): 387-397. 
Walstad, J.D., Anderson, R.F. and Stambaugh, W.J. (1970). Effect of environmental conditions on 2 species of muscardine fungi (Beauveria bassiana and Metarhizium anisopliae). J. Invert. Pathol. 16: 221-226.

Wikardi, E. A. (1983). Testing a virus and fungus for controlling Oryctes rhinoceros (Coleoptera, Scarabaeidae). Internal Report of Ballittri (Bogor Research Institute for Industrial Crops) in Indonesia, 16 pp.

Zimmermann, G. (1982). Effect of high temperatures and artificial sunlight on conidia of Metarhizium anisopliae. J. Invert. Pathol. 40: 36-40. 